\title{
Immune priming and clearance of orally acquired RNA viruses
}

\section{in Drosophila}

Juan A. Mondotte ${ }^{1}$, Valérie Gausson ${ }^{1}$, Lionel Frangeul ${ }^{1}$, Hervé Blanc ${ }^{1}$, Louis Lambrechts $^{2}$, Maria-Carla Saleh ${ }^{1 *}$

${ }^{1}$ Institut Pasteur, Viruses and RNA Interference Unit; Department of Virology; CNRS Unité Mixte de Recherche 3569, 75724 Paris Cedex 15, France.

${ }^{2}$ Institut Pasteur, Insect-Virus Interactions Group, Department of Genomes and Genetics; CNRS Unité Mixte de Recherche 2000, 75724 Paris Cedex 15, France.

*corresponding author: carla.saleh@pasteur.fr 


\section{Abstract}

Immune responses in insects are differentially triggered depending on the infection route used by the pathogen. In most studies involving Drosophila melanogaster and viruses, infection is done by injection while oral infection, probably the most common route of viral entry in nature, remains unexplored. Here we orally infect adults and larvae from wild-type and RNAi mutant flies with different RNA viruses. We find that, in contrast with what is observed following virus injection, oral infections initiated at larval or adult stages are cleared in adult flies. Virus elimination occurs despite a larger infectious dose than for injected flies and evidence of viral replication. RNAi mutant flies suffer extra mortality relative to wild-type flies following oral infection but they can also eliminate the virus, implying that RNAi is not essential for viral clearance and that other immune mechanisms act during oral infections. We further show that information of infection by RNA viruses acquired orally leaves a trace under a DNA form, which confers protection against future reinfection by the same virus. Together, this work presents evidence of clearance and immune priming for RNA viruses in insects and challenges the current view of antiviral immunity in insects. 
Host-pathogen interactions prompt selective pressures that drive the evolution of survival strategies for both organisms ${ }^{1,2}$. Of great consequence, the infection route taken by pathogens determines host adaptation by triggering differential immune responses ${ }^{3,4}$. The outcome of such hostpathogen interactions is highly variable and ranges from deleterious infections with lethal or permanent damage to completely innocuous infections. For example, an acute viral infection is typically characterized by a rapid onset of disease, high viral replication rates, and the production of large number of viral progeny. The infection is transient and is limited either by the death of the host or the active elimination (clearance) of the pathogen by the host immune system. On the other hand, a persistent infection results in long-lasting production of viral progeny that is tolerated by the host ${ }^{5}$.

Drosophila is a well-known model to study insect host-virus interaction and Drosophila $\mathrm{C}$ virus (DCV) is its most studied natural pathogen, a positivesense RNA virus belonging to the Dicistroviridae family. Most studies involving DCV have been performed by viral injections; while oral infection, probably the most frequent route of infection for this enterovirus, has largely been unexplored ${ }^{6}$. In general, DCV injection causes complete mortality within 3 to 13 days, depending on the viral dose and the fly genetic background ${ }^{7,8,9}$, 10, 11. Although virus injection successfully identified pathogen virulence factors and host defense mechanisms, injecting the virus bypasses the host natural protection barriers.

Different methods for oral infection in Drosophila have been described: exposing first-instar larvae to virus particles, or feeding adult flies with a mix of food and pathogen solution ${ }^{6}$. Oral infections activate different responses such 
as the Toll pathway ${ }^{12}$ and the nutrient responsive Extracellular signalRegulated Kinase (ERK) pathway ${ }^{13,14}$.

The small interfering RNA (siRNA) pathway is currently considered as the major antiviral pathway of insects because it controls infection by a wide range of viruses, including RNA and DNA viruses $8,9,15,16,17,18$. The siRNA pathway is triggered by double-stranded RNA (dsRNA) molecules such as viral replicative intermediates. This dsRNAs is recognized and cleaved by Dicer-2 (Dcr-2) into 21-nt viral siRNAs (vsiRNAs). Once produced, vsiRNAs guide the sequence-specific recognition and cleavage of viral RNAs by Argonaute 2 (Ago-2 $)^{19}$. In addition, RNA viruses produce viral-derived DNA (vDNA) molecules through the activity of endogenous retrotransposons, and this vDNA amplifies the RNAi-mediated antiviral immune response through the production of new vsiRNAs ${ }^{20,21}$. In Drosophila, however, studies that have established the key role of the siRNA pathway have always relied on injections and not oral infections.

In this study, we performed viral oral infections in larvae and adult wildtype flies as well as in flies deficient for the siRNA pathway. The results uncover a mechanism of clearance and immune priming for RNA viruses in Drosophila, and widen the current view of antiviral immunity in insects. 


\section{Results}

Oral virus infections are less virulent than injections

To compare the outcome of virus infection acquired orally vs. injection, and the role of RNAi in both conditions, we infected wild-type $w^{1118}, A g o-2^{-/-}$, and $D c r-2^{-/-}$flies by two different routes: injecting the viruses directly into the thorax or through the gut by feeding the virus to the fly (oral infection). We used the positive-sense RNA viruses DCV, Cricket Paralysis Virus (CrPV) and Flock House Virus (FHV).

When flies were infected by an intrathoracic injection of 50 tissueculture infectious dose $50 \%\left(\mathrm{TCID}_{50}\right)$ units per fly (Fig. 1a-c and Supplementary Fig. 1a), the three viruses caused an acute infection that inevitably resulted in the death of all flies in less than 9 days. As previously demonstrated $^{9,15}, 16,17,18,22$, Ago- $2^{-/-}$and $D c r-2^{-/-}$flies deficient for the siRNA pathway, are more sensitive to the infection as they succumb faster (Fig. 1ac). Viral loads were significantly higher in Ago-2 $2^{-/-}$flies than in wild-type flies at early but not at later times post infection (Supplementary Fig. 1b).

To investigate the role of the siRNA pathway in the antiviral response during an oral infection, Ago- $2^{-/}$and $D c r-2^{--}$flies were infected with DCV, CrPV, FHV or mock infected, and survival of the mutant flies was compared to that of the $w^{1118}$ flies. To verify the amount of virus ingested and rule out any effect of uncontrolled variation in feeding, we measured the amount of infectious virus in individual flies after different oral exposure times $(1 \mathrm{~h}, 8 \mathrm{~h}$, $16 \mathrm{~h}$ ) and compared it to the inoculum size of injections (Supplementary Fig. 2). Following oral exposure, regardless of exposure time, the infectious virus dose was significantly higher than following injection of $50 \mathrm{TCID}_{50}$ units per 
fly. Moreover, the measured viral input after oral exposure was consistent between flies across experiments and across exposure times. This confirms that oral exposure is a reliable route of experimental infection, allowing the dissection of antiviral immune responses under a more natural setting.

Less than $10 \%$ mortality was observed in $w^{1118}$ flies 15 days after oral infection with DCV, CrPV and FHV (Fig. 1d-f), consistent with previous studies $^{12,23,24}$. In contrast, 20-50\% mortality was observed for Ago-2/- and $D c r-2^{-/}$flies. While $w^{1118}$ flies started dying from 7 days post infection (dpi), virus-induced mortality in $\mathrm{Ago}-2^{-/-}$and $\mathrm{Dcr}-2^{-/-}$flies was observed as early as 2-3 dpi.

Altogether, these results indicate that a viral infection initiated orally is less virulent than intrathoracic injection in both $w^{1118}$ and RNAi-deficient flies, despite exposure to a larger infectious dose. Of note, virulence encompasses all fitness costs of infection ${ }^{25}$ and we only considered pathogen-induced mortality in the context of this study.

\section{Both wild-type and RNAi mutant flies clear the virus after oral infection}

Because the majority of flies fed with DCV survive until 15 dpi (90\% of $w^{1118}, 60 \%$ of $A g o-2^{-/}$and $70 \%$ of $D c r-2^{-/}$flies, Fig. 1d), we monitored the viral loads during the course of the infection. We found that viral titers decrease in $w^{1118}$ dropping at $15 \mathrm{dpi}$ to levels close to the detection threshold (Fig. 2a-b and Supplementary Fig. 3a-c - see methods section for details about calculation of mean-centered titers accounting for experimental variation). In both $A g o-2^{-/-}$and $D c r-2^{-/-}$flies, viral infection resulted in significantly increased mortality (Fig. 1d) and higher viral loads overall (Fig. 2a-b) than $w^{1118}$ controls, 
however these flies also display a drop in viral titers by 15 dpi. In agreement with these observations, the average viral prevalence (percentage of infected flies) decreased from $100 \%$ at 1 dpi to only $20 \%$ of $w^{1118}, 35 \%$ Ago-2-/- and $40 \%$ of $\mathrm{Dcr}-2^{-/-}$flies at $15 \mathrm{dpi}$ (Fig. 2c). These results suggest that during an oral infection with DCV, flies are capable to clear the infection in an RNAiindependent manner.

To rule out the possibility that the virus was not replicating at the early times of infection, we used a DCV suspension inactivated by UV irradiation (DCV UV) and we followed viral RNA by RT-PCR at 1 and 3 dpi. While viral RNA production at $1 \mathrm{dpi}$ in flies infected with the untreated virus was readily detected, flies infected with DCV UV showed a very weak PCR amplification product at $1 \mathrm{dpi}$ and the viral RNA was no longer detectable by $3 \mathrm{dpi}$ (Supplementary Fig. 3d). Furthermore, we deep-sequenced small RNAs from DCV orally infected $w^{1118}$ flies at 3, 6 and 15 dpi (Fig. 2d). As mentioned above, vsiRNAs arise from viral dsRNA replication intermediates. A high amount of vsiRNAs was detected at $6 \mathrm{dpi}$, but this number dropped close to the background at $15 \mathrm{dpi}$ due to the lack of virus replication at this time point. Taken together, these results confirm that during oral infections: $(i)$ the virus is replicating, as showed by the abundant presence of vsiRNAs covering both positive and negative strands of the DCV genome; (ii) the RNAi machinery is functional; and (iii) the virus is eliminated despite evidence of active replication.

Next, we asked whether viral clearance was a common response to viral oral infections in Drosophila. We analysed viral titers at different time points during a CrPV oral infection. Consistent with the increased 
susceptibility to infection of $\mathrm{Ago}-2^{-/-}$and $\mathrm{Dcr}-2^{-/-}$flies (Fig. 1e), an increase in viral replication was observed when compared to $w^{1118}$ flies (Fig. 3a-b and Supplementary Fig. 4a-c). Viral prevalence analysis (Fig. 3c) showed again an elimination of the virus at later times (at $15 \mathrm{dpi}$, less than $5 \%$ for $w^{1118}, 15 \%$ for $\mathrm{Ago-} 2^{-/-}$and none for $\mathrm{Dcr}-2^{-/-}$flies, were positive for $\mathrm{CrPV}$ on average). This result confirms that Drosophila is able to clear an oral viral infection even in the absence of the antiviral activity of the siRNA pathway.

Finally, we investigated the outcome of an oral infection with FHV, a virus that is not a natural Drosophila pathogen. Ago- $2^{-/-}$and $D c r-2^{-/}$flies were more susceptible to FHV oral infection than $w^{1118}$ flies (Fig. 1f) and displayed higher viral titers (Fig. 3d-e and Supplementary Fig. 4d-f). However, we noticed that at 15 dpi over $85 \%$ of $w^{1118}$ flies, $100 \%$ of $A g o-2^{-/-}$flies and $95 \%$ of $D c r-2^{-/}$flies were still FHV-positive (Fig. 3f). Thus, although an oral infection with the non-natural pathogen $\mathrm{FHV}$ is not cleared, $w^{1118}$ and $\mathrm{RNAi}$ mutant flies still control the burden of infection and survive to an orally delivered virus.

Infection initiated orally at larval stage is only cleared at adult stage

Since DCV is a natural pathogen of Drosophila and it is possible to initiate an infection by feeding the larvae with a viral suspension, we investigated viral clearance under these conditions. Drosophila eggs laid overnight in a regular rearing medium were contaminated with a DCV stock (Fig. 4a). When adult flies emerged, female flies were selected to monitor their survival and viral accumulation (Fig. $4 \mathrm{~b}-\mathrm{d}$ ). We observed that $w^{1118}$, Ago$2^{-/-}$and $D c r-2^{-/-}$flies started to die 2 to 4 days post emergence (dpe) (Fig. 4b). 
However, Ago-2-/ and Dcr-2-/ flies showed an increased death rate with about $50 \%$ and $20 \%$ mortality at 15 dpe, respectively. This increased susceptibility was also reflected in a higher viral accumulation over time (Fig. 4c-d). However, viral titers of $w^{1118}$ and RNAi mutant flies decreased over time (Fig. 4c-d and Supplementary Fig. 5a-c) indicating that the virus is cleared at the adult stage of flies infected orally as larvae. We measured viral prevalence over time and found that even though most flies were infected at $1 \mathrm{dpe}, \mathrm{DCV}$ prevalence decreased over time, reaching about 30\% for $w^{1118}, 60 \%$ for Ago$2^{-/}$and $45 \%$ for $D c r-2^{-/-}$flies at 15 dpe (Fig. 4e). In addition, we used singlestranded quantitative PCR (ssqPCR) directed to the negative strand of the viral RNA to assess viral replication ${ }^{26}$. Negative single-stranded viral RNA was detected at 3 dpe and subsequently decreased until 15 dpe both for $w^{1118}$ and Ago-2 $2^{-/}$flies (Supplementary Fig. 5d), indicating that in flies coming from infected larvae the virus is actively replicating at early times after adult emergence. Indeed, we observed a 1,000-fold decrease in the number of vsiRNAs in $w^{1118}$ flies collected at 15 dpe compared with those collected at 3 dpe (Fig. 4f). These findings indicate that a DCV oral infection initiated at the larval stage can be cleared at the adult stage.

We also found that the infection causes a significant decrease in larvato-pupa survival in both $w^{1118}$ and Ago-2-2 flies (Supplementary Fig. 5e) and that $A g o-2^{-/}$pupae showed a higher viral accumulation than $w^{1118}$ pupae (Supplementary Fig. 5f). A decrease in the pupa-to-adult survival after DCV infection was also observed (Supplementary Fig. 5g). DCV infected $w^{1118}$ pupae showed vsiRNAs covering the entire viral genome, indicating that the 
virus is actively replicating and that the RNAi machinery is functional (Supplementary Fig. 5h).

Altogether, the results show that wild-type and RNAi mutant flies clear a DCV oral infection initiated at the larval stage only when they reach the adult stage. They also indicate that the RNAi-independent antiviral mechanisms allowing viral clearance are developmental stage-specific.

\section{Infection initiated orally at larval stage leaves a viral DNA form in the adult}

Recently, we showed that adult flies infected with different RNA viruses produce vDNA molecules, through the activity of endogenous retrotransposons ${ }^{20,21}$. Since we observed that DCV orally infected flies are capable to clear a viral infection, we asked whether a vDNA would be produced during an oral infection. We extracted DNA and RNA from individual $w^{1118}$ adult flies from infected larvae at 3 and 15 dpe (Fig. 5a,b). At 3 dpe, when most of the flies are positive for DCV RNA, we detected DCV vDNA in $40 \%$ of them (Fig. 5a). At 15 dpe, when most of the flies have already cleared the virus and are negative for DCV RNA, we detected vDNA in $70 \%$ of them, even in the absence or viral replication (Fig. 5b). Therefore, an oral infection initiated at the larval stage leaves a trace under a DNA form in the adult, even after virus clearance.

vDNA serves as a template for the synthesis of new siRNAs that will boost the RNAi-mediated antiviral response. This amplification of the RNAi response can be mediated by $5^{\prime}$-monophosphorylated (pRNA) or 5'triphosphorylated (tripRNA) siRNAs ${ }^{20,27}$. To investigate if the vDNA form present in adult flies infected as larvae was producing pRNA and/or tripRNAs, 
we performed deep sequencing of whole adult flies at 15 dpe. These flies were negative for virus replication but positive for vDNA. The RNA fraction used to generate the library was treated with a 5' polyphosphatase enzyme to transform tripRNAs into pRNAs and the results compared to an untreated library that excludes tripRNAs. We used C. elegans libraries as a positive control for the detection of tripRNAs. We observed an enrichment of 22-ntlong small RNAs in C. elegans treated libraries (representing both pRNAs and tripRNAs) when compared to untreated libraries (excluding tripRNAs), indicating the production of secondary tripRNAs (Fig. 5c). In flies that cleared the virus and acquired a vDNA form, we could not detect a difference between treated and untreated libraries (Fig. 5d). The large size distribution of small RNAs (from 20 to $27 \mathrm{nt}$ ) is evocative of viral RNA degradation as these flies actively cleared the virus. Further studies are needed to address the role of the vDNA left in adult flies as a trace of infection during the larval stage.

Adult flies orally infected at larval stage show increased tolerance to future reinfections

A growing number of studies support the premise that invertebrates that have previously encountered a pathogen appear to be protected upon secondary exposure to the same pathogen, a concept known as immune priming $^{28,29,30,31,32}$. To explore if larval exposure to DCV protects against subsequent reinfection, we monitored survival of $w^{1118}$ adult flies emerged from DCV orally infected larvae after DCV challenge either by oral infection or by injection (Fig. 6a). Following challenge by oral infection, DCV priming at the larval stage did not affect adult mortality (Fig. 6b). We also measured a 
similar viral load in the DCV primed (DCV/DCV) flies and the control (Mock inf./DCV) flies (Supplementary Fig. 6a). However, following challenge by injection, DCV priming at the larval stage protected adult flies (Fig. 6c). This protection against future lethal infection is dependent on an intact RNAi pathway, as $\mathrm{Ago}-2^{-/-}$and $\mathrm{Dcr}-2^{-/-}$flies died at the same rate whether mock challenged or DCV challenged. Viral loads between mock infected or DCV challenged flies remain unchanged (Supplementary Fig. 6b-d). Increased survival despite unchanged viral loads suggests a change in tolerance, which is defined as the regression of health (survival) by pathogen load (viral titer) ${ }^{33 \text {, }}$ 34. Indeed, we observed a change in the slope of the tolerance curves between DCV primed and control flies (Fig. 6d), suggesting that tolerance is the mechanism underlying immune priming. Finally, we tested whether the protection observed is virus-specific. We challenged adult flies coming from DCV orally infected larvae with CrPV or FHV. We did not observe protection for these reciprocal cross-infections (Supplementary Fig. 6e-g) indicating that the priming and the protection observed are virus and sequence specific.

These results show that previous exposure to DCV by oral infection at the larval stage reduces the susceptibility to a lethal viral challenge later in life with the same virus providing evidence of transstadial immune priming.

\section{Discussion}

The transmission route taken by pathogens to infect their hosts has a profound impact on the outcome of the infection ${ }^{3,12,35,364 .}$

In this work, we performed viral oral infections in larvae and adult Drosophila flies using different model RNA viruses. We found that delivering 
virus orally produces a less virulent infection than by injection. In addition, virus was undetectable in most of the orally infected flies after 15 days, showing that oral infections can be cleared despite active virus replication. Oral infections are probably the most usual route of infection in nature and flies have presumably evolved specific mechanisms to control infections in their gut. In most DCV oral infections the virus localizes and is controlled locally at the gut level. However, previous studies showed that in DCV orally infected flies, the virus was detected in the intestinal visceral muscles ${ }^{13}$ and in the fat body ${ }^{12}$. This indicates that the virus could be capable of breaching the midgut barrier, reaching the haemolymph, spreading systemically and eventually killing the fly. That would explain why some degree of virulence is observed when wild-type or RNAi mutant flies are orally infected with different RNA viruses.

Ago- $2^{-/-}$and $\mathrm{Dcr}-2^{-/-}$flies were more susceptible after an oral challenge and displayed higher viral titers than wild-type flies, but unexpectedly, approximately $60 \%$ of infected flies were still alive 15 days after infection. The virus was still present in some flies, indicating that RNAi is not essential to reach persistent infections initiated by oral infection. Furthermore, the virus was undetectable in some flies, showing that viral clearance is possible in the absence of a functional RNAi machinery. In addition, Habayeb et al. $^{37}$ discovered that Nora Virus, a picorna-like virus that lacks cytopathic effects when injected into flies, can either give rise to persistent infections or be cleared independently of the RNAi pathway. These results challenge the current view that the siRNA pathway is essential during a viral infection and suggest that following oral infections other antiviral mechanisms might be 
involved to control and eliminate the virus. Conversely, we hypothesize that RNAi is essential to control systemic infections established once the virus breaches the gut barrier and colonizes other tissues. That would help to explain why injection of DCV, CrPV or FHV directly into the haemolymph, is lethal.

We also observed viral clearance in adult flies coming from DCV infected larvae. In these flies we also found evidence of viral replication preceding the clearance. Controversially, it was published that in adult flies coming from DCV infected larvae the virus is not actively replicating ${ }^{26}$. The authors performed ssqPCR to detect negative-strand viral RNA (indicative of viral replication) but did not detect it. In our work, we detected negative-sense RNA using the same primers as the mentioned study. We conjecture that authors performed the measurement several days after emergence of adult flies, when the virus was already cleared.

We found that adult flies coming from DCV infected larvae produced a vDNA that remains even after viral clearance. It is tempting to speculate that the vDNA could protect flies from future reinfections. Studies in bees showed that a percentage of the insect population carries a segment of the Israeli Acute Paralysis Virus in their genome and this subpopulation is virus resistant $^{38}$. DCV vDNA could be actively involved in antiviral immunity, increasing resistance or tolerance to new viral infections in the same organism. In addition, this vDNA could be occasionally inherited and confer trans-generational protection but additional studies are necessary to prove this hypothesis. 
Finally, we found that adult flies coming from DCV primed larvae display increased tolerance to a subsequent lethal challenge with the same virus. This immune priming is RNAi-dependent and virus specific. Interestingly, Longdon et al. ${ }^{39}$ published that flies injected with a sublethal dose of DCV are not protected against a subsequent DCV lethal injection. This lack of protection indicates that the oral infection route is probably essential to effectively prime the immune response and that injection of virus cannot prime the system. These results, together with a study in Lepidoptera showing protection against a DNA virus ${ }^{40}$, indicate that the phenomenon of immune priming in insects encompasses viral infections.

In conclusion, using different modes of infection (oral vs. injection) reveals a substantial contrast in the antiviral immune response. We demonstrated that following oral exposure: $(i)$ viral infections persist or are cleared at the adult stage; (ii) RNAi is not essential to clear viral infections or to reach persistence; (iii) infection leaves a trace in the host under a vDNA form even after clearance; (iv) flies orally exposed to the virus as larvae are protected from lethality during reinfection by injection as adults; and $(v)$ transstadial immune priming is RNAi-dependent, virus and sequence-specific. By revealing the fundamental difference between virus injection and oral infection, probably the most common route of infection in nature, our work opens a new avenue for the study of antiviral immune responses in insects and other invertebrates. 


\section{Methods}

No statistical methods were used to predetermine sample size. Experiments were not randomized, and the co-authors were not blinded to allocation during experiments and assessments of the results.

\section{Fly Strains and Husbandry}

The fly lines used were the following:

$w^{1118} ;+;+$

$w^{1118} ;+;$ Ago-2 $2^{414}$

$w^{1118} ;$ Dcr-2 ${ }^{\mathrm{L811fs}} ;+$

Fly stocks are on the same genetic background than $w^{1118}$ and harbour the sensitive allele of Pastrel 3L:7350895 (Thr). Flies were maintained on a standard cornmeal diet (Bloomington) at a constant temperature of $25^{\circ} \mathrm{C}$. All fly lines were cleaned of possible chronic infections (viruses and Wolbachia) as described previously ${ }^{41}$. In addition, fly stocks were analysed by RT-PCR with pairs of primers specific for CrPV, DAV, DXV, DCV, FHV, and NoraV.

\section{Virus Production and Titration}

DCV, CrPV and FHV stocks were prepared in $w^{1118}$ flies. Flies were injected intrathoracically with $500 \mathrm{TCID}_{50}$ per fly. When mortality started flies were anesthetized and squashed in PBS (3 flies per $100 \mu$ of PBS). The extract was frozen at $-80^{\circ} \mathrm{C}$, thawed and centrifuged for $15 \mathrm{~min}$ at $15,000 \mathrm{xg}$ at $4^{\circ} \mathrm{C}$. The supernatant was recovered and filtered to eliminate bacteria, aliquoted and stored at $-80^{\circ} \mathrm{C}$. Stocks were tittered in S2 cells and titers were 
measured by end-point dilution method and expressed as TCID 50 . Titers for all viruses stocks were around $5 \times 10^{9} \mathrm{TCID}_{50} / \mathrm{ml}$.

To quantify viral load in pupae and flies, individual pupae or flies were squashed in $100 \mu \mathrm{l}$ of PBS at the indicated time points and tittered.

To inactivate DCV for UV irradiation, $250 \mu$ of viral stocks were exposed to UV irradiation in 24 wells plate (area, $1 \mathrm{~cm}^{2}$ ) on melting ice (to prevent heating effects and evaporation) for $15 \mathrm{~min}$. The UV source (UVItec, Cambridge, United Kingdom) emitted UV at $312 \mathrm{~nm}$. Samples were titrated after exposure to verify viral inactivation.

\section{Viral Infections}

Injection: 4-6 days after emergence female flies were injected intrathoracically using a nanoject (Nanoject II apparatus; Drummond Scientific) with $50 \mathrm{~nL}$ of a viral suspension in $10 \mathrm{mM}$ Tris, $\mathrm{pH}$ 7. An injection of the same volume of 10 $\mathrm{mM}$ Tris, $\mathrm{pH} 7$ served as a mock-infected control. Infected flies were kept at $25^{\circ} \mathrm{C}$ and changed to fresh vials every 2 days.

Larva infection: 25 female and 25 male flies were put in a clean media tube during 16h. Next day, adult flies were discarded and embryos were infected by adding $100 \mu \mathrm{l}$ of viral stock in top of them. The following day, embryos were re-infected with the same amount of virus. Developed pupae were carefully removed with a brush, washed three times with PBS and transferred to a new clean media tube. Flies were collected after adult emergence to performed different tests.

Adult oral infection: 4-6 days after emergence female flies were starved during $8 \mathrm{~h}$ in an empty tube. Then, flies were transferred to an empty tube containing 
a Whatman filter paper in the bottom embedded in a mix of viral stock in PBS ( $10 \%$ viral stock, $35 \%$ sucrose and $2 \%$ of blue dye). After $16 \mathrm{~h}$, only the flies having a blue-belly (corresponding to blue dye in the gut due to ingestion) were placed in clean media tubes. Flies were transferred to clean media tubes every 2 days during the course of the experiment.

\section{Survival Assays}

Adult flies: survival of infected flies (adults infected by injection or orally; adults coming from infected larvae) was measured daily by counting the number of dead flies in each test tube. Fly mortality at day 1 was attributed to damage invoked by injection and/or manipulation procedure, and excluded from further analyses.

Larva and pupa survival: male and female flies were transferred to egg-laying cages made of grape juice plates with yeast paste on top, during 16h. Next day, embryos were carefully collected and placed in regular media tubes. Once pupae formed, the number of pupae was scored to calculate survival. Pupa to adult survival was calculated on the number of emerged adult flies from a known pupae number.

\section{RNA Extraction and Library Production}

For each time point of infection analysed, total RNA was extracted from 15 flies or pupae. For each sample, 19-31 nt small RNAs were purified from a $15 \%$ acrylamide/bisacrylamide (37.5:1), $7 \mathrm{M}$ urea gel as described previously $^{42}$. Purified small RNAs were used for library preparation using the NEBNext Multiplex Small RNA Library Prep for Illumina (New England 
Biolabs) with the $3^{\prime}$ adapter from Integrated DNA Technologies (linker 1) and in-house-designed indexed primers. Libraries were diluted to $4 \mathrm{nM}$ and sequenced using the NextSeq 500 High-Output Kit v2 (Illumina) (75 cycles) on a NextSeq 500 sequencer (Illumina). Reads were analysed with in-house Perl scripts.

\section{5 '-triphosphorylated small RNA sequencing}

Two $\mu g$ of total RNA extracted with TRIzol were treated with 20 units of RNA 5' Polyphosphatase enzyme (Epicentre), 30 minutes at $37^{\circ} \mathrm{C}$. Treated samples were extracted with acid phenol: chloroform, $\mathrm{pH} 4.5$ (Ambion) and precipitated with $15 \mu \mathrm{g}$ Glycoblue (Thermo Fisher Scientific), 0.1 volumes of 3M Sodium acetate and 2.5 volumes of ice cold $100 \%$ ethanol overnight at $-80^{\circ} \mathrm{C}$. After centrifugation $\left(30 \mathrm{~min}, 4^{\circ} \mathrm{C}\right)$ and $70 \%$ ethanol wash, RNA pellets were resuspended in $20 \mathrm{ul}$ of water and proceed to the small RNA library production.

\section{Bioinformatics Analysis of Small RNA Libraries}

The quality of fastq files was assessed using graphs generated by FastQC (www.bioinformatics.babraham.ac.uk/projects/fastqc/). Using cutadapt (https://cutadapt.readthedocs.io/en/stable/), low-quality bases and adaptors were trimmed from each read. Only reads with acceptable quality (phred score 20) were retained. FastQC generated a second set of graphs on the fastq files created by cutadapt. Reads were mapped to genomes using bowtie1 (https://sourceforge.net/projects/bowtie-bio/files/bowtie/1.0.0/) with the $-\mathrm{v} 1$ (one mismatch between the read and its target). bowtie1 generates 
results in sam format. All sam files were analysed by the samtools package (http://samtools.sourceforge.net/) to produce bam-indexed files. To analyze these bam files, graphs were generated using custom $R$ scripts (https://www.r-project.org/) and the Bioconductor (https://www.bioconductor.org/) Rsamtools and Shortreads libraries.

\section{RNA extractions and RT-PCR}

Virus containing samples were extracted with TRIzol (Invitrogen). Before RTPCR analysis, samples were treated with DNase I (Roche). cDNAs were produced with SuperScript II Reverse Transcriptase (Invitrogen) with random hexamer primers. PCRs were performed with a Green Taq DNA Polymerase (GenScript).

\section{ssq-PCR}

Total RNA was extracted from individual flies with TRIzol, then $1 \mu \mathrm{g}$ total RNA was treated with DNase I (Roche). The cDNA was prepared by reverse transcription with SuperScript II Reverse Transcriptase (Invitrogen) using DCV-tag primer (to selectively amplify the negative strand of DCV) or random hexamer primers (to target Rp49). Roche Universal Sybr Green Master Mix (Rox) was used for ssq-PCR. Quantification was normalized to that of mRNA encoding the endogenous ribosomal protein Rp49. Data were calculated using the $\triangle \triangle \mathrm{Cq}$ method to compute relative gene expression. DCV-R, DCVtag and Tag primers (from Stevanovic et al. ${ }^{26}$ ) and Rp49 103-F and Rp49 207-R primers (see sequence in DNA oligonucleotides list provided in Supplementary Information) were used. A 7900HT Fast Real-Time PCR 
System (Applied Biosystems) was used with the following program: $95^{\circ} \mathrm{C}$ for 5 min, followed by 40 cycles of $95^{\circ} \mathrm{C}$ for $15 \mathrm{~s}, 60^{\circ} \mathrm{C}$ for $30 \mathrm{~s}$, followed by a melt analysis to confirm that only the expected products were amplified.

\section{DNA extraction for vDNA PCR analysis}

DNA was simultaneously isolated with total RNA from individual flies according to the TRIzol manufacturer's instructions. PCR analysis for detection of DCV vDNA was performed using DreamTaq DNA polymerase with the primers indicated in each Figure (see sequence in DNA oligonucleotides list provided in Supplementary Information). PCR products were sequenced to confirm that products being amplified corresponded to the targeted sequence.

\section{Statistical Analyses}

Infection prevalence was analysed as a binary variable by logistic regression. Infectious viral titers were compared by analysis of variance (ANOVA) after $\log _{10}$-transformation+1. qPCR results were analysed by ANOVA after $\log _{10^{-}}$ transformation of normalized values. Larval survival and pupal survival were analysed by a weighted full-factorial ANOVA of the percentage of pupation and percentage of adult emergence, respectively. Time point was considered an ordinal variable. Full-factorial models initially included interactions up to the second order. Because of missing combinations, the interaction between experiment and time point was omitted from the analysis of oral infection of adult flies by DCV, CrPV and FHV, and from the analysis of oral infection of larvae by DCV. For the same reason, the interaction between genotype and 
time point was omitted from the analysis of DCV-injected adult flies. Full models were subsequently reduced to minimal adequate models by backward elimination of non-significant terms in a stepwise fashion. Effects were considered statistically significant if their $p$ value was $<5 \%$.

Because all the experiments were repeated multiple times, uncontrolled variation between experiments was accounted for in the statistical analyses. In addition, to visually correct for the experiment effect in the graphical representations, the raw data for viral titers and survival were adjusted for variation between experiments. Adjusted values were obtained using a statistical procedure that removes all the variation that can be explained by the experimental replicates and adjusts their means to be equal. This procedure consists of plotting the residuals from a one-way ANOVA as a function of experiment. This procedure transforms the raw values into their deviation from the experiment mean, and the resulting adjusted values are centered around zero. The mathematical explanation is as follows. Let a be an observed value and $\log _{10}(a)$ the $\log _{10}$-transformed value. When the observed values are estimated with the model that only includes the experiment effect, the estimate of $\log _{10}(a)$ is $\log _{10}(\hat{a})$ where â is the experiment average. Residuals are defined as the difference between observed values and estimated values and represent variation unexplained by the model. They are calculated as $\log _{10}(a)-\log _{10}(\hat{a})$, which can be written as $\log _{10}(a / a ̂)$. Therefore, residuals are the observed values scaled by their experiment average, expressed on a $\log _{10}$ scale.

The comparison of survival curves was done by a log-rank (Mantel-Cox) test. 
All statistical analyses were performed in JMP v10.0.2 (www.jmp.com) or

Prism version 6.00 (www.graphpad.com).

\section{Data availability}

The data that support the findings of this study are available from the corresponding author upon request. In-house codes are also available at any time upon request to the authors. Small RNA libraries are publicly available in the Sequence Read Archive with accession codes PRJNA396531 and PRJNA473322.

\section{References}

1. Stern, A. \& Sorek, R. The phage-host arms race: shaping the evolution of microbes. Bioessays 33, 43-51 (2011).

2. Obbard, D.J. \& Dudas, G. The genetics of host-virus coevolution in invertebrates. Curr Opin Virol 8, 73-78 (2014).

3. Martins, N.E., Faria, V.G., Teixeira, L., Magalhaes, S. \& Sucena, E. Host adaptation is contingent upon the infection route taken by pathogens. PLoS Pathog 9, e1003601 (2013).

4. Chambers, M.C., Jacobson, E., Khalil, S. \& Lazzaro, B.P. Thorax injury lowers resistance to infection in Drosophila melanogaster. Infect Immun 82, 4380-4389 (2014).

5. Goic, B. \& Saleh, M.C. Living with the enemy: viral persistent infections from a friendly viewpoint. Curr Opin Microbiol 15, 531-537 (2012).

6. Mondotte, J.A. \& Saleh, M.C. Antiviral Immune Response and the Route of Infection in Drosophila melanogaster. Adv Virus Res 100, 247-278 (2018).

7. Jousset, F.X., Plus, N., Croizier, G. \& Thomas, M. [Existence in Drosophila of 2 groups of picornavirus with different biological and serological properties]. C R Acad Sci Hebd Seances Acad Sci D 275, 3043-3046 (1972).

8. Galiana-Arnoux, D., Dostert, C., Schneemann, A., Hoffmann, J.A. \& Imler, J.L. Essential function in vivo for Dicer-2 in host defense against RNA viruses in drosophila. Nat Immunol 7, 590-597 (2006). 
9. van Rij, R.P. et al. The RNA silencing endonuclease Argonaute 2 mediates specific antiviral immunity in Drosophila melanogaster. Genes Dev 20, 2985-2995 (2006).

10. Dostert, C. et al. The Jak-STAT signaling pathway is required but not sufficient for the antiviral response of drosophila. Nat Immunol 6, 946953 (2005).

11. Merkling, S.H. et al. The epigenetic regulator G9a mediates tolerance to RNA virus infection in Drosophila. PLoS Pathog 11, e1004692 (2015).

12. Ferreira, A.G. et al. The Toll-dorsal pathway is required for resistance to viral oral infection in Drosophila. PLoS Pathog 10, e1004507 (2014).

13. Xu, J. et al. ERK signaling couples nutrient status to antiviral defense in the insect gut. Proc Natl Acad Sci U S A 110, 15025-15030 (2013).

14. Sansone, C.L. et al. Microbiota-Dependent Priming of Antiviral Intestinal Immunity in Drosophila. Cell Host Microbe 18, 571-581 (2015).

15. Zambon, R.A., Vakharia, V.N. \& Wu, L.P. RNAi is an antiviral immune response against a dsRNA virus in Drosophila melanogaster. Cell Microbiol 8, 880-889 (2006).

16. Li, H., Li, W.X. \& Ding, S.W. Induction and suppression of RNA silencing by an animal virus. Science 296, 1319-1321 (2002).

17. Bronkhorst, A.W. et al. The DNA virus Invertebrate iridescent virus 6 is a target of the Drosophila RNAi machinery. Proc Natl Acad Sci U S A 109, E3604-3613 (2012).

18. Mueller, S. et al. RNAi-mediated immunity provides strong protection against the negative-strand RNA vesicular stomatitis virus in Drosophila. Proc Natl Acad Sci U S A 107, 19390-19395 (2010).

19. Bronkhorst, A.W. \& van Rij, R.P. The long and short of antiviral defense: small RNA-based immunity in insects. Curr Opin Virol 7, 19-28 (2014).

20. Goic, B. et al. RNA-mediated interference and reverse transcription control the persistence of RNA viruses in the insect model Drosophila. Nat Immunol 14, 396-403 (2013).

21. Goic, B. et al. Virus-derived DNA drives mosquito vector tolerance to arboviral infection. Nat Commun 7, 12410 (2016).

22. Nayak, A. et al. Cricket paralysis virus antagonizes Argonaute 2 to modulate antiviral defense in Drosophila. Nature Structural \& Molecular Biology 17, 547-U541 (2010). 
23. Jousset, F.X. \& Plus, N. [Study of the vertical transmission and horizontal transmission of "Drosophila melanogaster" and "Drosophila immigrans" picornavirus (author's transl)]. Ann Microbiol (Paris) 126, 231-249 (1975).

24. Wong, Z.S., Brownlie, J.C. \& Johnson, K.N. Impact of ERK activation on fly survival and Wolbachia-mediated protection during virus infection. J Gen Virol 97, 1446-1452 (2016).

25. Alizon, S., Hurford, A., Mideo, N. \& Van Baalen, M. Virulence evolution and the trade-off hypothesis: history, current state of affairs and the future. $J$ Evol Biol 22, 245-259 (2009).

26. Stevanovic, A.L. \& Johnson, K.N. Infectivity of Drosophila C virus following oral delivery in Drosophila larvae. J Gen Virol 96, 1490-1496 (2015).

27. Tassetto, M., Kunitomi, M. \& Andino, R. Circulating Immune Cells Mediate a Systemic RNAi-Based Adaptive Antiviral Response in Drosophila. Cell 169, 314-325 e313 (2017).

28. Sadd, B.M. \& Schmid-Hempel, P. Insect immunity shows specificity in protection upon secondary pathogen exposure. Curr Biol 16, 1206-1210 (2006).

29. Little, T.J. \& Kraaijeveld, A.R. Ecological and evolutionary implications of immunological priming in invertebrates. Trends Ecol Evol 19, 58-60 (2004).

30. Kurtz, J. \& Franz, K. Innate defence: evidence for memory in invertebrate immunity. Nature 425, 37-38 (2003).

31. Tate, A.T. A general model for the influence of immune priming on disease prevalence. Oikos 126, 350-360 (2017).

32. Pham, L.N., Dionne, M.S., Shirasu-Hiza, M. \& Schneider, D.S. A specific primed immune response in Drosophila is dependent on phagocytes. Plos Pathogens 3 (2007).

33. Raberg, L., Sim, D. \& Read, A.F. Disentangling genetic variation for resistance and tolerance to infectious diseases in animals. Science 318, 812-814 (2007).

34. Louie, A., Song, K.H., Hotson, A., Thomas Tate, A. \& Schneider, D.S. How Many Parameters Does It Take to Describe Disease Tolerance? PLoS Biol 14, e1002435 (2016). 
35. Behrens, S. et al. Infection routes matter in population-specific responses of the red flour beetle to the entomopathogen Bacillus thuringiensis. BMC Genomics 15, 445 (2014).

36. Gupta, V. et al. The route of infection determines Wolbachia antibacterial protection in Drosophila. Proc Biol Sci 284 (2017).

37. Habayeb, M.S., Ekengren, S.K. \& Hultmark, D. Nora virus, a persistent virus in Drosophila, defines a new picorna-like virus family. J Gen Virol 87, 3045-3051 (2006).

38. Maori, E., Tanne, E. \& Sela, I. Reciprocal sequence exchange between nonretro viruses and hosts leading to the appearance of new host phenotypes. Virology 362, 342-349 (2007).

39. Longdon, B., Cao, C., Martinez, J. \& Jiggins, F.M. Previous exposure to an RNA virus does not protect against subsequent infection in Drosophila melanogaster. PLoS One 8, e73833 (2013).

40. Tidbury, H.J., Pedersen, A.B. \& Boots, M. Within and transgenerational immune priming in an insect to a DNA virus. Proc Biol Sci 278, 871-876 (2011).

41. Merkling, S.H. \& van Rij, R.P. Analysis of resistance and tolerance to virus infection in Drosophila. Nat Protoc 10, 1084-1097 (2015).

42. Gausson, V. \& Saleh, M.C. Viral small RNA cloning and sequencing. Methods Mol Biol 721, 107-122 (2011).

Acknowledgements: We thank members of the Saleh lab, especially Vanesa Mongelli, for fruitful discussions. Marie-Anne Felix and Lise Frezal for help with 5 'triphosphorylated sRNAs detection in C. elegans. This work was supported by the European Research Council (FP7/2013-2019 ERC CoG 615220) to MCS and the French Government's Investissement d'Avenir program, Laboratoire d'Excellence Integrative Biology of Emerging Infectious Diseases (grant ANR-10-LABX-62-IBEID) to MCS and LL. JAM was supported by AXA Research fund. LL received support from the City of Paris Emergence(s) program in Biomedical Research, the European Union's Horizon 2020 research and innovation program under ZikaPLAN grant 
agreement No 734584, and the Agence Nationale de la Recherche (grants ANR-16-CE35-0004 and ANR-17-ERC2-0016-01).

Author contribution: Conceptualization: J.A.M. and M.-C.S.; Investigation: J.A.M., V.G., L.F., and H.B.; Software: L.F.; Formal analysis: J.A.M., L.F., and L.L.; Writing: J.A.M., L.L., and M.-C.S.; Funding acquisition: J.A.M., L.L., and M.-C.S.

Competing financial interests: The authors declare no competing financial interests.

Materials \& Correspondence: carla.saleh@pasteur.fr to whom correspondence and material requests should be addressed. 


\section{Figure Legends:}

Figure 1. Wild-type and RNAi mutant flies are less susceptible to viruses delivered by oral route.

a, b, c $w^{1118}, A g o-2^{-/-}$and $D c r-2^{-/-}$adult flies were intrathoracically injected with $50 \mathrm{TCID}_{50}$ units of $\operatorname{DCV}(\mathbf{a}), \operatorname{CrPV}(\mathbf{b})$ or FHV (c) and survival was measured daily. Two independent experiments with three biological replicates of $\mathrm{n}=10$ flies each per condition were analysed. $\mathbf{d}, \mathbf{e}, \mathbf{f}, w^{1118}, A g o-2^{-/}$and $D c r-2^{-/}$adult flies were orally infected with a mix of sucrose and blue-dye containing $5 \times 10^{8} \mathrm{TCID}_{50}$ units of $\mathrm{DCV}(\mathbf{d})$, CrPV (e) or FHV (f) and survival was measured daily. Four independent experiments with three biological replicates of $n=15$ flies each per condition were analysed. Within each fly genotype, infection with any of the three viruses significantly increased mortality $(p<0.05)$. Error bars indicate SEM. Survival curves were compared by log-rank (Mantel-Cox) test.

\section{Figure 2. Adult flies clear orally acquired DCV independently of RNAi.}

a, b, Mean viral titers over time are plotted expressed as $\log _{10} T C{ }^{2} D_{50} /$ fly from a representative experiment (a) and as mean-centered titers for all experiments combined after correction for experimental variation (b). For the representative experiment $(\mathrm{a}) \mathrm{n}=75$ flies for $w^{1118}, \mathrm{n}=75$ for $A g o-2^{-/-}$and $\mathrm{n}=$ 75 for $D c r-2^{-/}$were analysed. Mean-centered titers in (b) represent $n=500$ flies for $w^{1118}, \mathrm{n}=220$ for Ago-2 $2^{-/}$and $\mathrm{n}=320$ for $D c r-2^{-/-}$from at least three independent experiments per genotype. Stars indicate statistical significance of pairwise comparisons by a two-tailed Student's $t$-test with $w^{1118}$ as the reference $\left({ }^{*} p \leq 0.05,{ }^{* *} p \leq 0.01,{ }^{* *} p \leq 0.001\right)$. The absence of star indicates lack of statistical significance $(p>0.05)$. Error bars indicate SEM. One unit on the mean-centered scale corresponds to a 10-fold difference on the original scale. c, Percentage of infected flies (prevalence) from (b) in $w^{1118}$ (open blue circles), Ago-2/- (open red squares) and $\mathrm{Dcr}-2^{-/-}$(open orange triangles). Circles, squares and triangles represent independent experiments for each time point. Open bars indicate the weighted mean per time point. Statistical significance of the time point effect indicated below the graph was obtained 
with logistic regression and likelihood-ratio test (the minimal adequate model is provided in the supplementary information). d, Profiles of $21 \mathrm{nt}$ small RNA reads that mapped to the DCV genome at 3, 6 and $15 \mathrm{dpi}$. Sense and antisense siRNAs are shown in orange and blue, respectively. Uncovered positions are in grey. The number of siRNAs for each strand is indicated in the right top corner of each profile. Data are representative of three independent experiments.

Figure 3. Control of oral viral infection is a common outcome for Drosophila viruses.

a-c, Viral titers and prevalence of CrPV orally infected flies. d-f, Viral titers and prevalence of FHV orally infected flies. Mean viral titers over time are plotted expressed as $\log _{10} \mathrm{TCID}_{50} /$ fly from a representative experiment (a,d) and as mean-centered titers for all experiments combined after correction for experimental variation (b,e). For the representative experiment in (a) $n=71$ flies for $w^{1118}, \mathrm{n}=71$ for $A g o-2^{-/-}$and $\mathrm{n}=72$ for $D c r-2^{-/-}$were analysed. For the representative experiment in (d) $n=90$ flies for $w^{1118}, \mathrm{n}=90$ for $\mathrm{Ago-} \mathrm{2}^{-/-}$and $\mathrm{n}$ $=89$ for $D c r-2^{-/-}$were analysed. Mean-centered titers in (b) represent $n=270$ flies for $w^{1118}, \mathrm{n}=290$ for $A g o-2^{-/-}$and $\mathrm{n}=165$ for $\mathrm{Dcr}-2^{-/-}$that were analysed for CrPV from at least three independent experiments. Mean-centered titers in (e) represent $\mathrm{n}=165 \mathrm{w}^{1118}, \mathrm{n}=165 \mathrm{Ago}-2^{-/-}$and $\mathrm{n}=165 \mathrm{Dcr}-2^{-/-}$flies that were analysed for FHV from two independent experiments. Stars indicate statistical significance of pairwise comparisons by a two-tailed Student's $t$-test with $w^{1118}$ as the reference $\left({ }^{*} p \leq 0.05,{ }^{* *} p \leq 0.01,{ }^{* * *} p \leq 0.001,{ }^{* * * *} p \leq\right.$ 0.0001). The absence of star indicates lack of statistical significance ( $p>$ 0.05). Error bars indicate SEM. One unit on the mean-centered scale corresponds to a 10-fold difference on the original scale. $\mathbf{c}, \mathbf{f}$, Percentage of $\mathrm{CrPV}(\mathbf{c})$ and FHV (f) infected flies (prevalence) from (b and $\mathbf{e}$ ) in $w^{1118}$ (open blue circles), $A g o-2^{-/-}$(open red squares) and $\mathrm{Dcr}-2^{-/}$(open orange triangles). Circles, squares and triangles represent independent experiments for each time point. Open bars indicate the weighted mean per time point. Statistical significance of the time point effect indicated below the graph was obtained 
with logistic regression and likelihood-ratio test (the minimal adequate model is provided in the supplementary information).

\section{Figure 4. DCV acquired at the larval stage is cleared at the adult stage.}

a, Schematic of the protocol. Male and female flies were put in a clean tube for $16 \mathrm{~h}$. The next day adult flies were discarded and embryos were infected by adding DCV viral stock. The following day, embryos were re-infected. After 6 to 7 days, developed pupae were removed with a brush, washed and transferred to a new tube with clean medium until adult fly emergence. b, Survival of $w^{1118}, A g o-2^{-/-}$and $D c r-2^{-/-}$adult flies from infected larvae was measured daily. At least five independent experiments with three biological replicates of $n=15$ flies each per condition were analysed. Error bars indicate SEM. Survival curves were compared by log-rank (Mantel-Cox) test. c, d, Mean viral titers over time are plotted expressed as $\log _{10} \mathrm{TCID}_{50} /$ fly from a representative experiment (c) and as mean-centered titers for all experiments combined after correction for experimental variation (d). For the representative experiment in (c) $\mathrm{n}=59$ flies for $w^{1118}, \mathrm{n}=55$ for Ago-2 $2^{-/-}$and $\mathrm{n}$ $=59$ for $D c r-2^{-/-}$were analysed. Mean-centered titers in (d) represent $n=395$ flies for $w^{1118}, \mathrm{n}=340$ for $A g o-2^{-/-}$and $\mathrm{n}=260$ for $D c r-2^{-/-}$from at least five independent experiments that were analysed per genotype. Stars indicate statistical significance of pairwise comparisons by a two-tailed Student's $t$-test with $w^{1118}$ as the reference $\left({ }^{*} p \leq 0.05,{ }^{* *} p \leq 0.01,{ }^{* * *} p \leq 0.001,{ }^{* * * *} p \leq\right.$ 0.0001). The absence of star indicates lack of statistical significance $(p>$ 0.05). Error bars indicate SEM. One unit on the mean-centered scale corresponds to a 10-fold difference on the original scale. e, Percentage of infected flies (prevalence) from (d) in $w^{1118}$ (open blue circles), Ago-2 ${ }^{-/}$(open red squares) and $D c r-2^{--}$(open orange triangles). Circles, squares and triangles represent independent experiments for each time point. Open bars indicate the weighted mean per time point. Statistical significance of the time point effect indicated below the graph was obtained with logistic regression and likelihood-ratio test (the minimal adequate model is provided in the supplementary information). f, Profiles of $21 \mathrm{nt}$ small RNA reads that mapped to the DCV viral genome at 3 and 15 dpe. Sense and antisense siRNAs are shown in orange and blue, respectively. Uncovered positions are in grey. The 
number of siRNAs for each strand is indicated in the right top corner of each profile. Data are representative of two independent experiments.

Figure 5. A vDNA form remains after viral clearance in orally infected flies.

a, b, Viral DNA (top panel) and viral RNA (bottom panel) were amplified by PCR and RT-PCR, respectively, from individual adult flies at 3 dpe (a) and 15 dpe (b) following DCV oral infection at the larval stage. To confirm the absence of viral replication at $15 \mathrm{dpe}$, two different pairs of primers were used for RT-PCR. Viral replication was considered active only when both pairs of primers produced an amplification product. Rp49 was used as housekeeping control. Data are representative of three independent experiments. Full uncropped scans for DNA gels are shown in Supplementary Figures 7 and 8. c, d, Size distribution of sense and antisense small RNA reads from $C$. elegans (Chromosome III; position 178600 to 179500) positive control for the RNA 5' Polyphosphatase treatment (c) and from flies that cleared DCV oral infection (d). White bars represent 5'-monophosphorylated small RNAs; grey bars represent 5'-monophosphorylated + 5'-triphosphorylated small RNAs. Data are representative of two independent experiments.

Figure 6. DCV orally infected flies are protected from future reinfections. a, Schematic of the protocol. $w^{1118}$ and RNAi mutant adult flies were orally infected at the larval stage and collected; 7 to 8 dpe flies were challenged either orally or by injection of $5 \mathrm{TCID}_{50}$ units of DCV per fly and their survival was monitored daily. $\mathbf{b}$, Survival curve of $w^{1118}$ adult flies after DCV challenge by feeding. Three independent experiments with three biological replicates of $\mathrm{n}=15$ flies each per condition were analysed. Error bars indicate SEM c, Survival curve of $w^{1118}, A g o-2^{-/-}$and $D c r-2^{-/}$adult flies after DCV challenge by injection. At least three independent experiments with three biological replicates of $\mathrm{n}=12$ flies each per condition were analysed. Error bars indicate SEM. Survival curves in (b) and (c) were compared by log-rank (Mantel-Cox) test. d, Disease tolerance curves for DCV/DCV and Mock inf./DCV flies, calculated as the regression of health (median survival time) by pathogen load $\left(\mathrm{TCID}_{50} / \mathrm{fly}\right)$. For Mock inf./DCV flies the slope of the tolerance curve $(-0.49 \pm$ 
$0.17)$ is significantly different from zero $(p=0.0229)$, whereas for DCV/DCV flies the slope $(-0.05 \pm 0.33)$ is not significantly different from zero $(p=$ 0.8793). Each data point represents an independent experiment and nine experiments were analysed. Disease tolerance curves were analysed by simple linear regression and ANOVA. DCV/DCV refers to primed/challenged flies, whereas Mock inf./DCV refers to unprimed/challenged flies. 
a

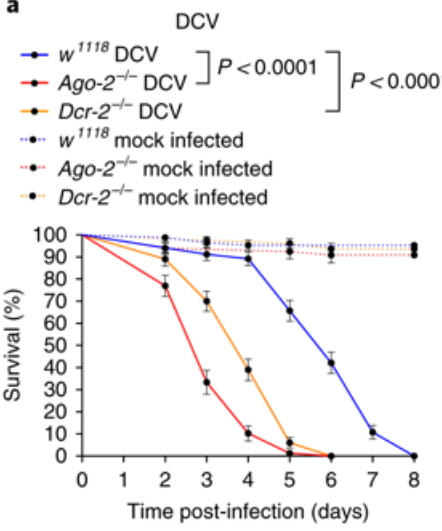

d

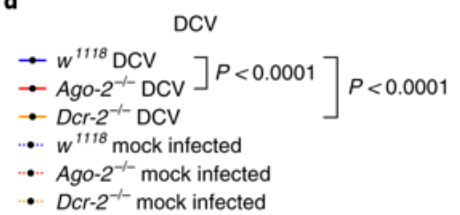

$\left.\left.\begin{array}{l}\rightarrow w^{1118} \mathrm{CrPV} \\ \rightarrow \text { Ago-2 } 2^{-1-} \mathrm{CrPV}\end{array}\right] P<0.0001\right] P<0.01$

.. $w^{1118}$ mock infected

- Ago-2 $2^{-1-}$ mock infected

- Dcr-2 $2^{-1-}$ mock infected

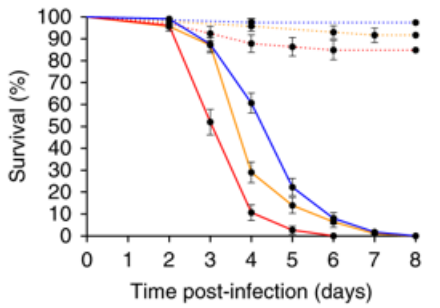

.

CrPV

$\left.\left.\because w^{1118} \mathrm{CrPV}\right] P<0.0001\right] P<0.0001$

- Dcr-2 $2^{-1-}$ CrPV

-. $w^{1118}$ mock infected

-. Ago-2 $2^{-1-}$ mock infected

- $D c r-2^{-1-}$ mock infected

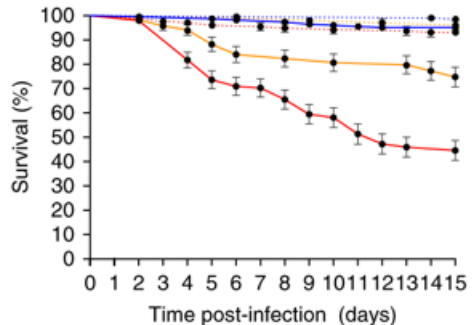

FHV

$\left.\left.\begin{array}{l}\rightarrow w^{1118} \mathrm{FHV} \\ \rightarrow \text { Ago- } 2^{-1} \mathrm{FHV}\end{array}\right] P<0.05\right]$ n.s.

$w^{1118}$ mock infected

-. Ago- $2^{-1-}$ mock infected

- Dcr-2 $2^{-1}$ mock infected

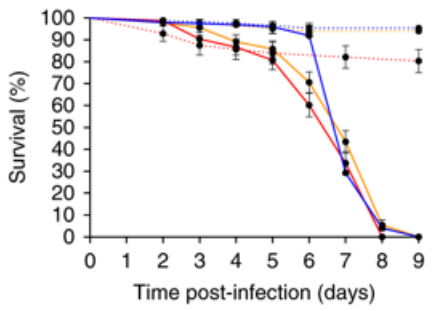

f
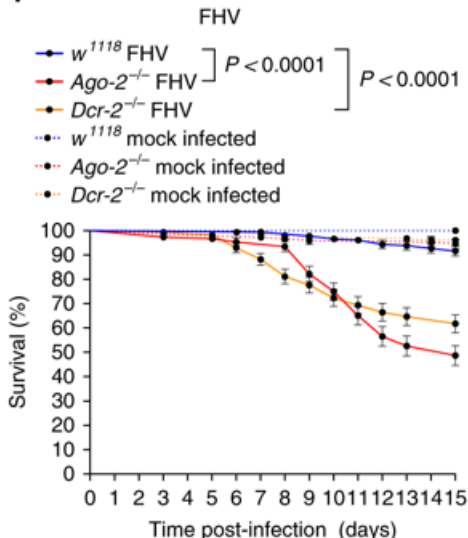

Fig. 1: Wild-type and RNAi mutant flies are less susceptible to viruses delivered orally. 

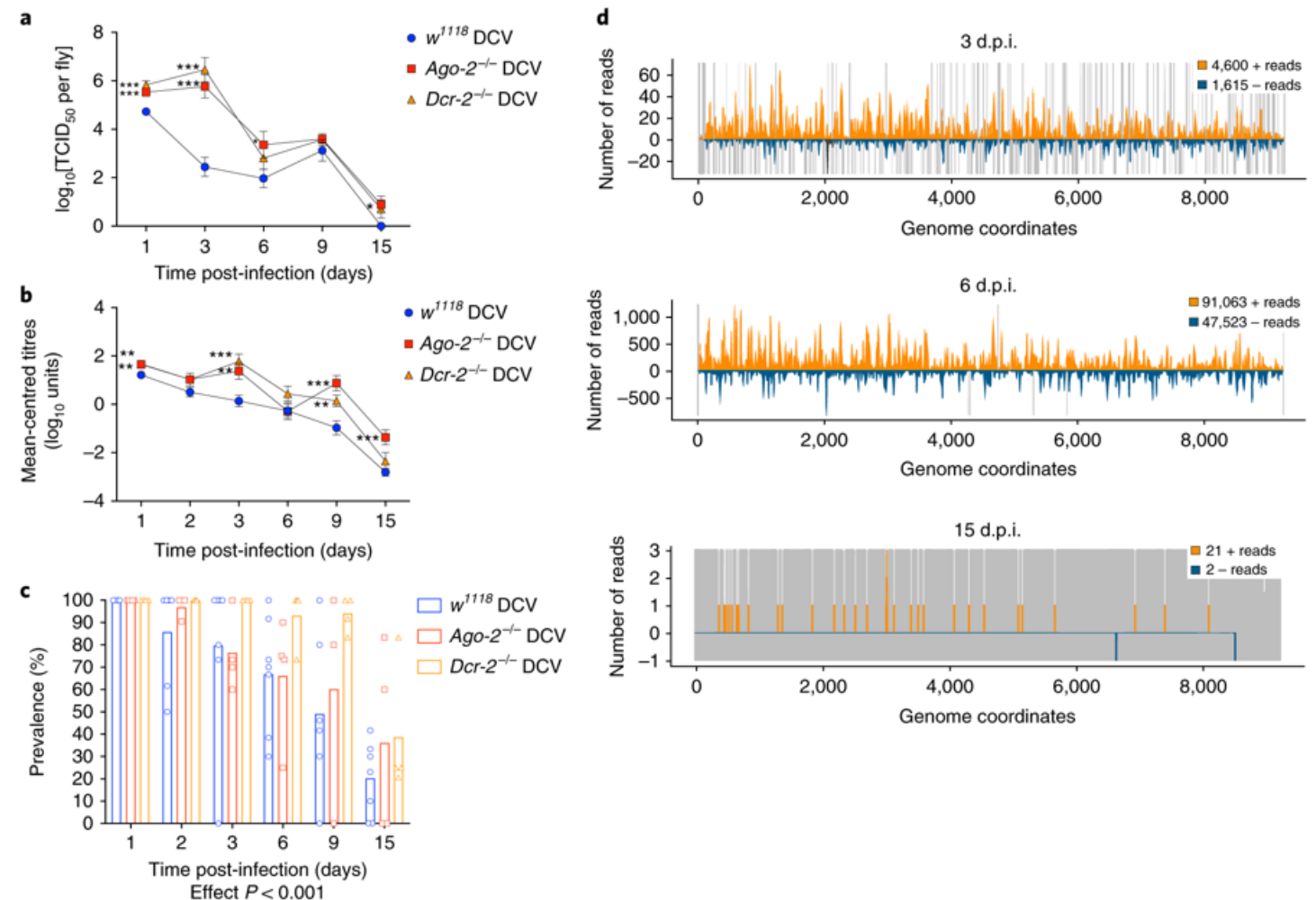

Fig. 2: Adult flies clear orally acquired DCV independently of RNAi. 

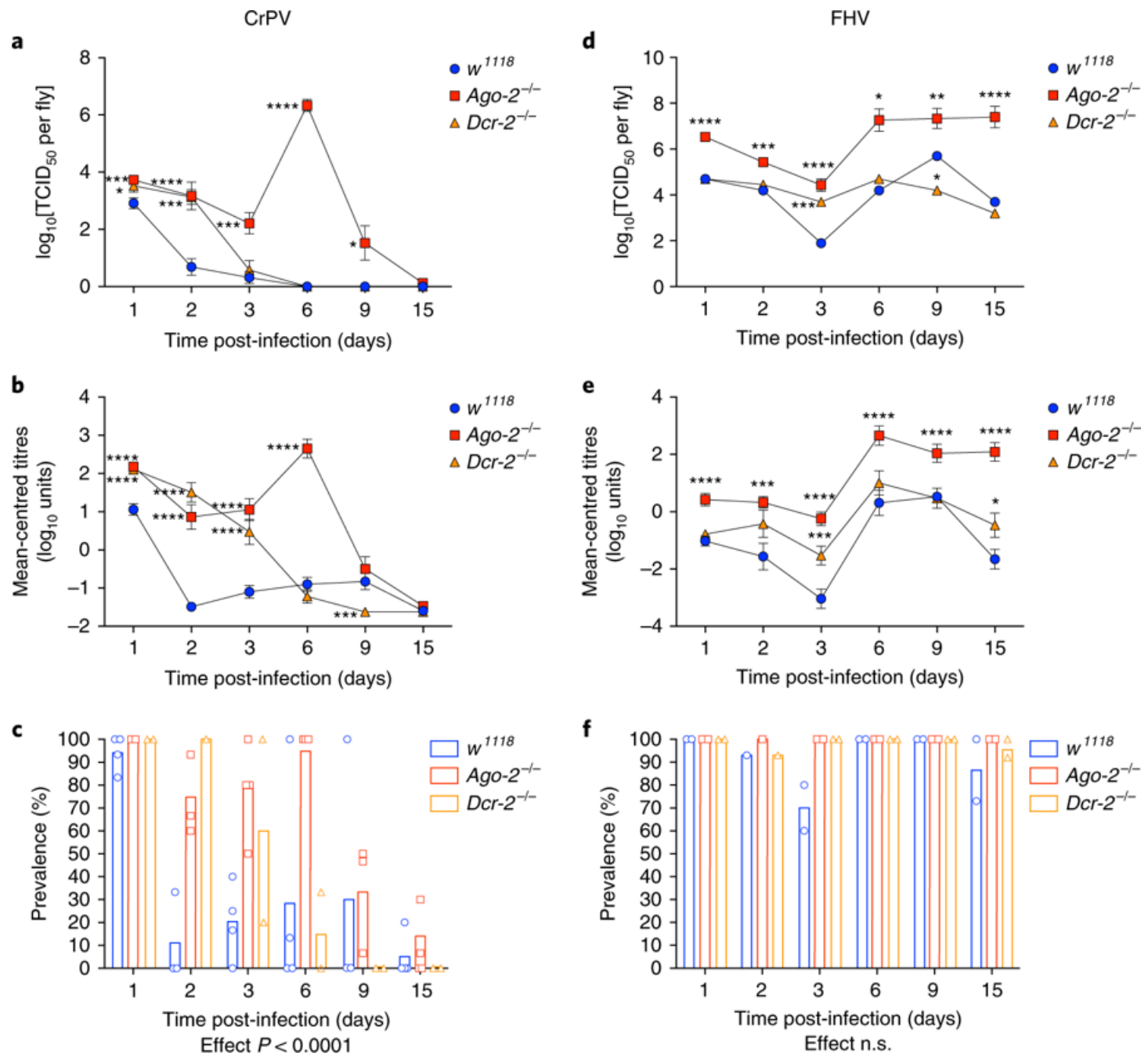

Fig. 3: Control of oral viral infection is a common outcome for Drosophila viruses. 

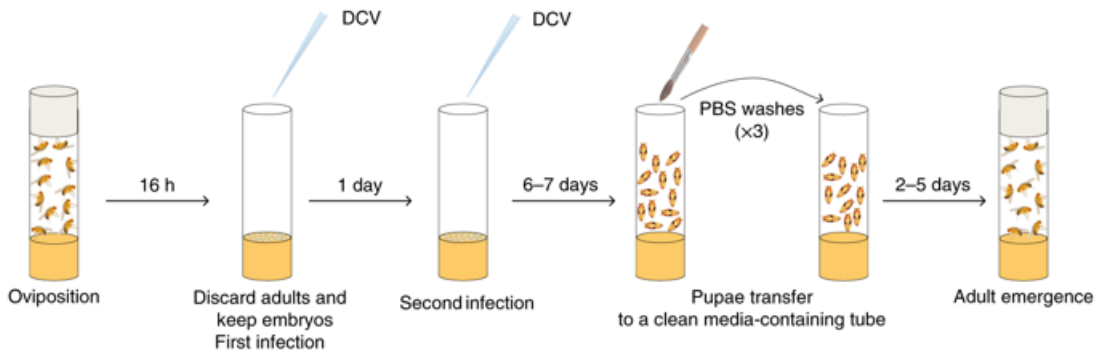

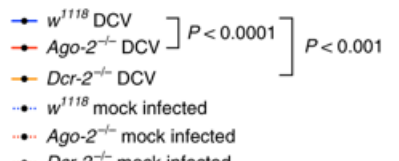

$$
\text { c }
$$

- $w^{1118} \mathrm{DCV}$

- $\mathrm{Ago}-2^{+-} \mathrm{DCV}$

$\triangle D C r-2^{--} \mathrm{DCV}$

- $w^{1118} \mathrm{DCV}$
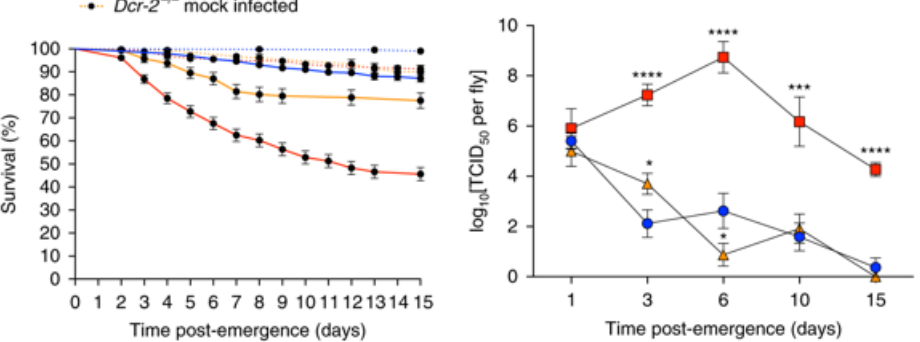

- $\mathrm{Ago}-2^{-1-} \mathrm{DCV}$

$\triangle D C r-2^{-1-} \mathrm{DCV}$
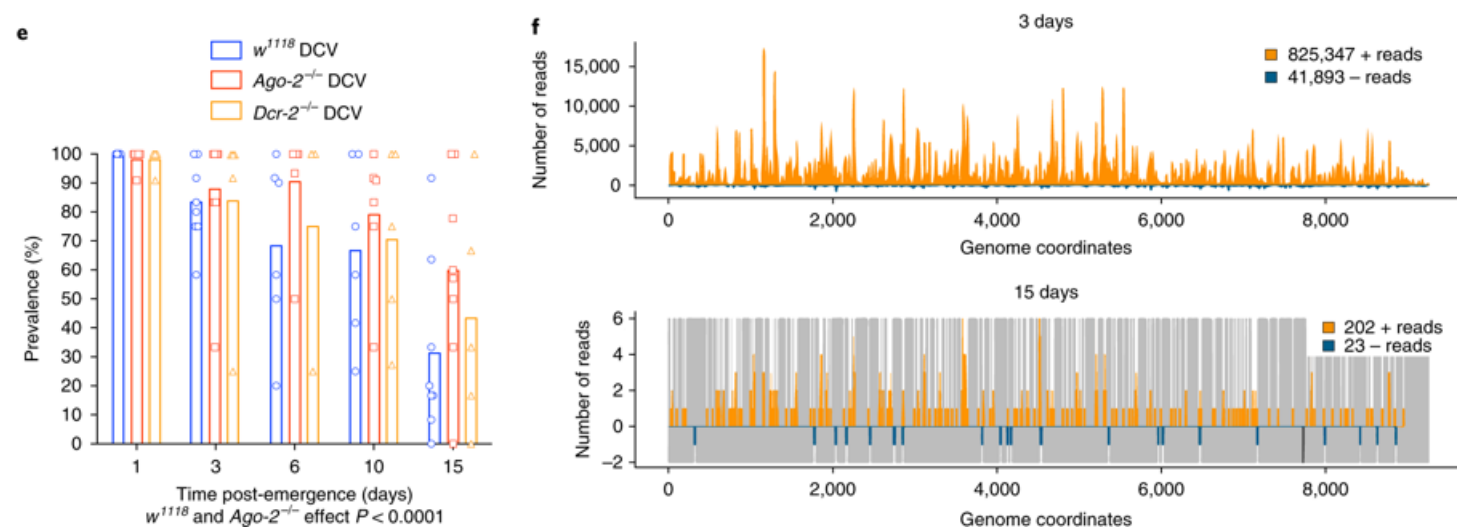

15 days

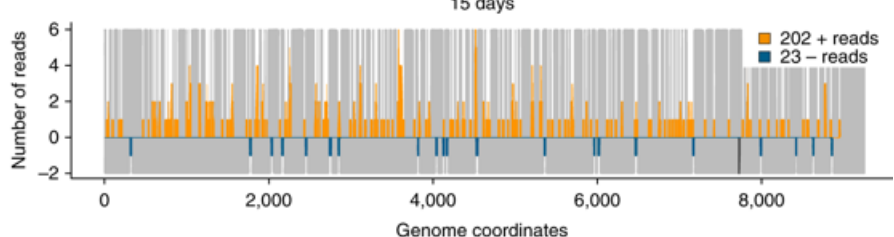

Fig. 4: DCV acquired at the larval stage is cleared at the adult stage. 
a
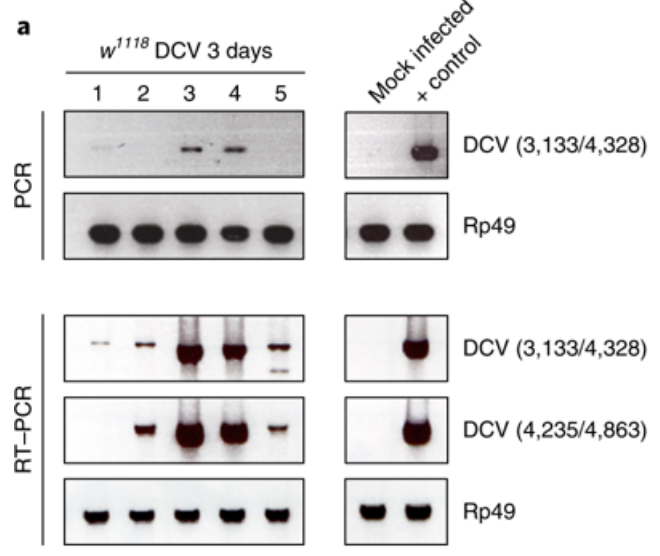

c

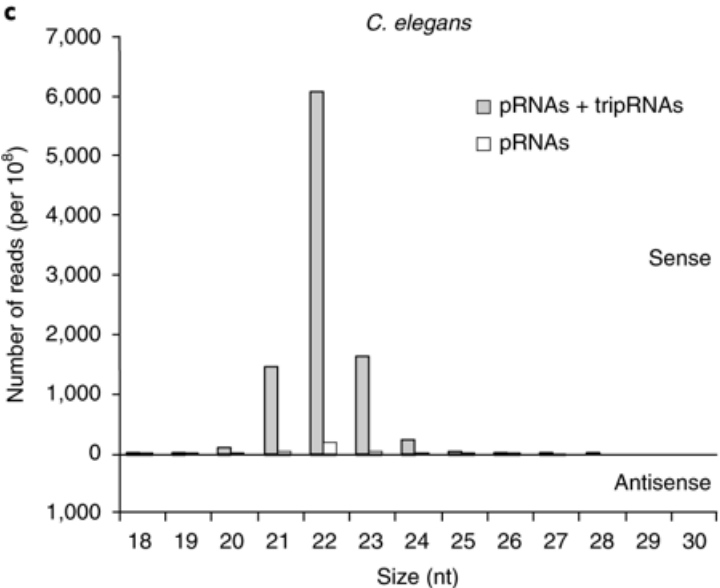

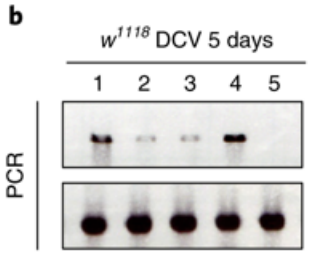
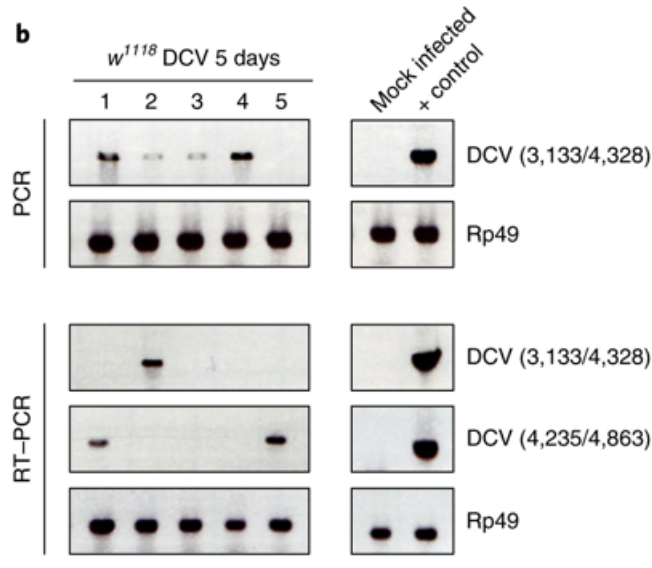

d

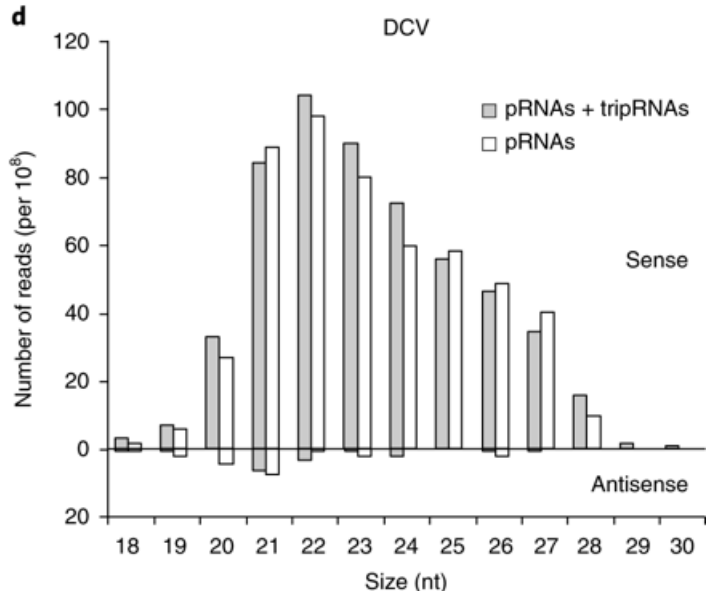

Fig. 5: A vDNA form remains after viral clearance in orally infected flies. 


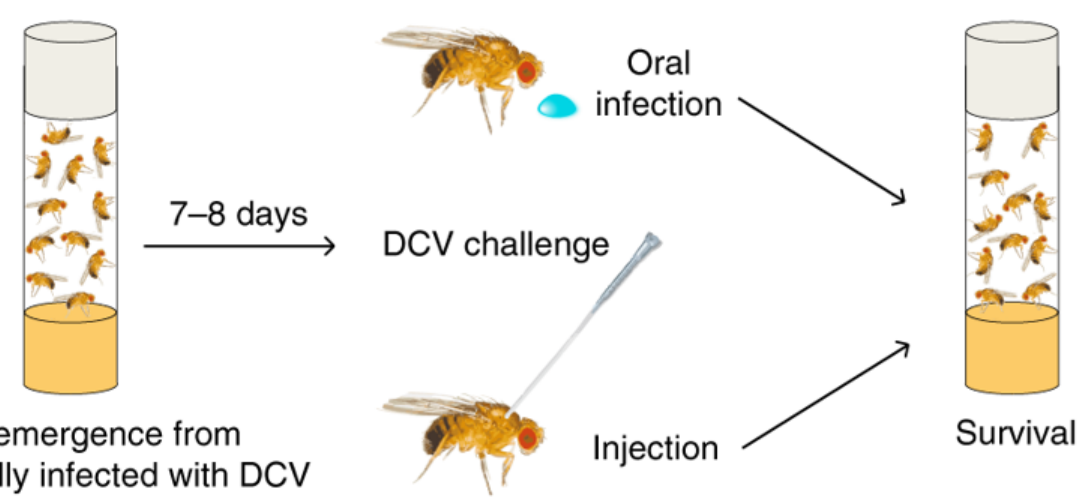
larvae orally infected with DCV
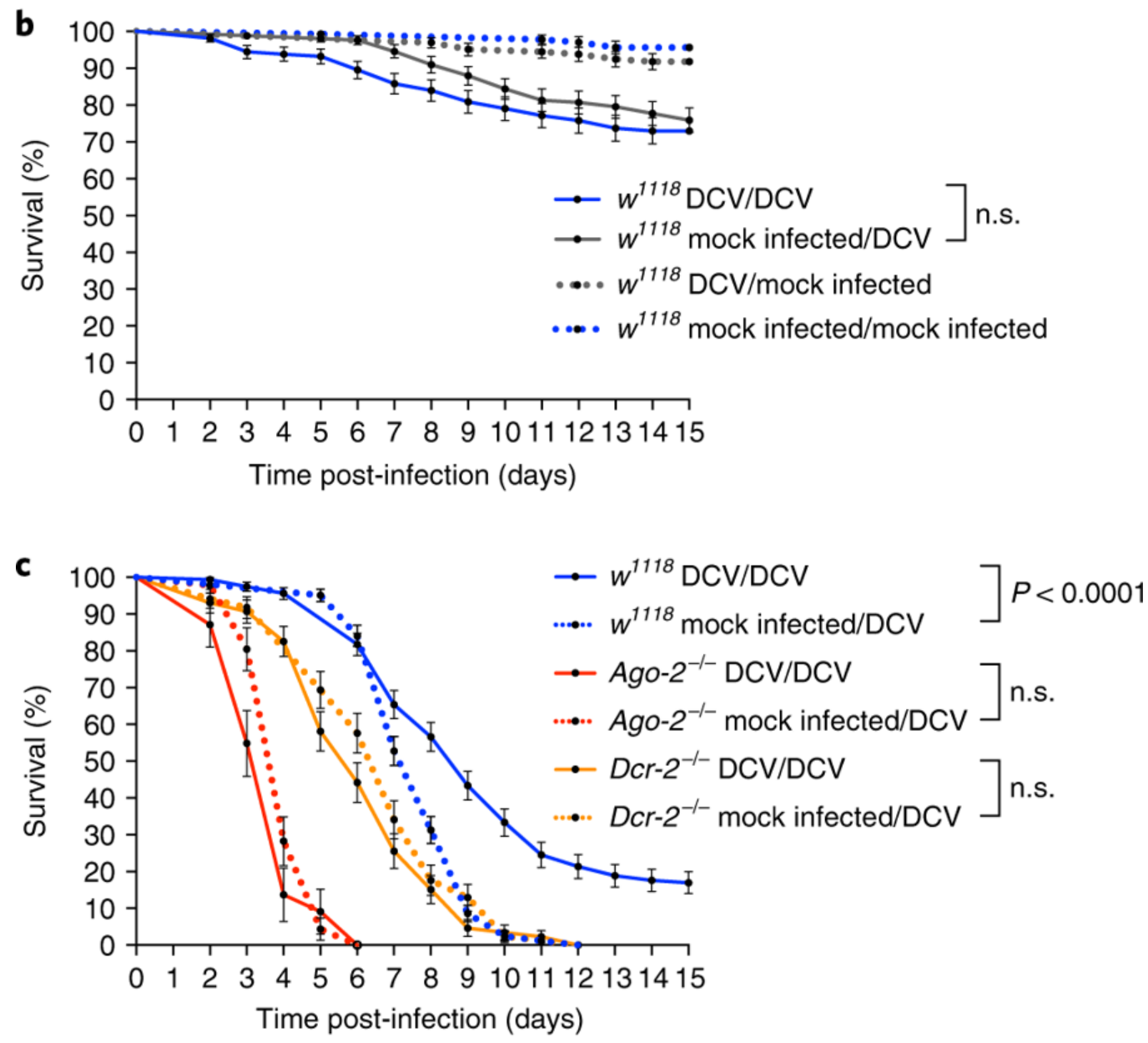

d

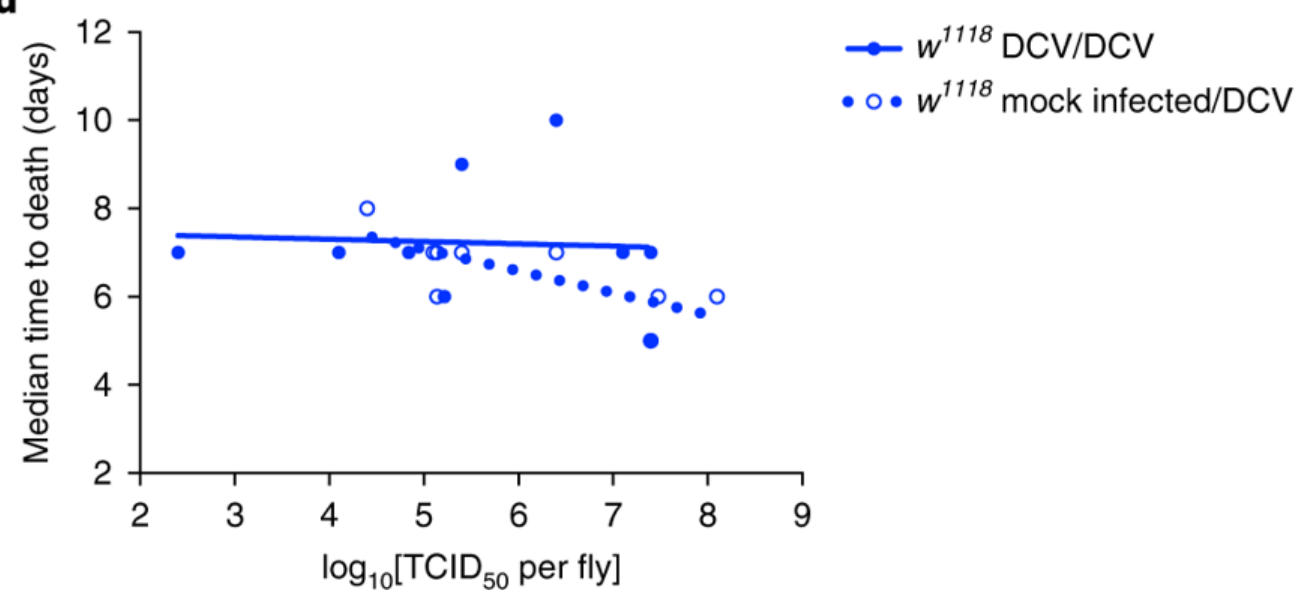


Fig. 6: Flies orally infected with DCV are protected from future reinfections. 